\title{
Activity-Independent Segregation of Excitatory and Inhibitory Synaptic Terminals in Cultured Hippocampal Neurons
}

\author{
Deanna L. Benson and P. Aryeh Cohen \\ Fishberg Research Center for Neurobiology, Mount Sinai School of Medicine, New York, New York 10029
}

\begin{abstract}
Cultured hippocampal neurons were used as a model system to address experimentally the spatial and temporal sequence leading to the appropriate sorting of excitatory and inhibitory synaptic terminals to different cellular target domains and the role of neural activity in this process. By using antibodies against glutamic acid decarboxylase 65 (GAD65) and synaptophysin, we examined the development and segregation of GABAergic and non-GABAergic synaptic terminals on single neurons. Electron microscopy confirmed that GAD65-labeled swellings observed using light microscopy corresponded to synaptic boutons. From the time at which GABAergic terminals first appeared, they developed at a more rapid rate on neuronal somata than non-GABAergic terminals did, such that by $18 \mathrm{~d}$ in culture, $60 \%$ of the total boutons on somata were GABAergic. By contrast, the majority $(70 \%)$ of boutons on dendrites were non-GABAergic. These data suggest that inhibitory synaptic
\end{abstract}

terminals are targeted preferentially to or maintained on cell somata at the expense of excitatory terminals. Interestingly, non-GABAergic terminals were not inhibited from forming synapses on cell somata, because in the absence of GABAergic terminals they attained the same total somatic terminal density seen in the presence of GABAergic terminals. Chronic blockade of neuronal activity did not affect the differential targeting of GABAergic and non-GABAergic axons; however, it did reduce the extent of dendritic arborization. Our findings support a two-step model for synaptic segregation whereby the majority of terminals is initially targeted in an activity-independent manner to the appropriate cellular domains, but an additional developmental mechanism serves to further restrict and refine the original synaptic distribution.

Key words: synaptogenesis; presynaptic terminal; hippocampus; neuronal culture; TTX; GABAergic
Excitatory and inhibitory synaptic terminals are differentially distributed on their postsynaptic target cells. On hippocampal and neocortical pyramidal cells, excitatory synapses outnumber inhibitory synapses, but they predominate on distal dendrites and dendritic spines (Gray, 1959; Andersen et al., 1966; Beaulieu and Colonnier, 1985). In contrast, inhibitory synapses predominate on cell somata and dendritic shafts and, although numerically fewer, are positioned near the site of action-potential generation to strongly influence neuronal output (Gray, 1959; Blackstad and Flood, 1963; Hamlyn, 1963; Andersen et al., 1964; Uchizono, 1968). Such spatial segregation of functionally distinct synapses is critical for normal integrative properties of synaptic neurotransmission (Rall, 1970). The sequence through which such functionally distinct types of synaptic terminals become properly sorted on their target neurons is unknown, nor is it known what factors play a role in regulating synaptic sorting, such as chemical or electrical signals.

Several lines of evidence indicate that axons in both vertebrates and invertebrates find their target areas by following pathways defined by gradients of chemical signals (Jessell, 1988; Glanzman et al., 1991; Broadie, 1994; Goodman, 1994; Tessier-Lavigne, 1994). The signaling molecules can be attractants or repellents, secreted by targets, or bound to substrates. Although some axonal

Received June 10, 1996; revised July 17, 1996; accepted July 24, 1996.

This research was supported by National Science Foundation Grant IBN-9419900 (D.L.B.). We thank Robert Woolley, Dariel L. S. Cobb, and Marina B. Rubin for their technical assistance, and Dr. G. W. Huntley for his comments on this manuscript.

Correspondence should be addressed to Dr. Deanna L. Benson, Fishberg Research Center for Neurobiology, Box 1065, Mount Sinai School of Medicine, One Gustave L. Levy Place, New York, NY 10029.

Copyright (C) 1996 Society for Neuroscience $0270-6474 / 96 / 166424-09 \$ 05.00 / 0$ growth cones may be directed precisely by specific signals to their final synaptic targets (Bate, 1976; Ready and Nicholls, 1979; Bastiani and Goodman, 1986; Zoran et al., 1990), many axons terminate in initially widespread arbors, which in response to competition from overlapping inputs are pruned and remodeled (Goodman and Shatz, 1993; Hall and Sanes, 1993). It is not known whether axons arising from excitatory and inhibitory neurons precisely target different cellular domains or whether they innervate their targets nonselectively and undergo subsequent rearrangement. Distinguishing between these two developmental strategies is a critical first step in determining the mechanisms underlying synaptic sorting.

One obvious way in which excitatory and inhibitory axon terminals might be distinguished from one another is by the different neurotransmitters they use. In the developing CNS, competition between afferents and the resultant highly ordered connections are often mediated by activity-dependent neurotransmitter release (Stryker and Harris, 1986; Shatz and Stryker, 1988; Fox et al., 1996). Thus, activity-dependent excitatory and inhibitory neurotransmission with subsequent interactions between different neurotransmitters and their specific receptors may play a role in the segregation of glutamatergic and GABAergic terminals to relatively separate cellular domains.

Cultured hippocampal neurons, which consist only of pyramidal (glutamatergic) neurons and GABAergic interneurons, provide a relatively simple system in which to examine the strategies used to sort excitatory and inhibitory synapses to different cellular domains (Benson et al., 1994; Craig et al., 1994). In culture, basketlike GABAergic terminals form on somata of pyramidal neurons, and non-GABAergic terminals form on dendritic spines, strongly suggesting that excitatory and inhibitory synapses segregate ap- 
propriately (Craig et al., 1993; Benson et al., 1994). Synapses identified by the immunocytochemical localization of synaptic vesicle proteins can be analyzed and quantified by light microscopy (Fletcher et al., 1991; Benson et al., 1994). Those synapses that are GABAergic can be identified by antibodies against glutamic acid decarboxylase 65 (GAD65) (Benson et al., 1994), an isoform of the GABA-synthesizing enzyme GAD, which is targeted to synaptic vesicles (Kaufman et al., 1991; Reetz et al., 1991; Shi et al., 1994).

In the present study, we used cultured hippocampal neurons to determine the spatial and temporal sequence through which GABAergic and non-GABAergic synaptic terminals become targeted differentially to their proper cellular domains, and we investigated the role of neural activity in initiating this process.

\section{MATERIALS AND METHODS}

Cultures. Hippocampi were dissected from 18-d-old, fetal Sprague Dawley rats, and cell cultures were prepared as described previously (Banker and Cowan, 1977; Goslin and Banker, 1991). Cells were dissociated by treatment with $0.25 \%$ trypsin for $15 \mathrm{~min}$ at $37^{\circ} \mathrm{C}$, followed by trituration through a fire-polished Pasteur pipette. The cells were plated at a density of 50,000 cells per $60 \mathrm{~mm}$ plastic Petri dish on poly-L-lysine-coated glass coverslips in minimum essential media (MEM) (Biotechnologies, Grand Island, NY) containing $10 \%$ horse serum. After the cells had attached (about $4 \mathrm{hr}$ ), the coverslips were transferred to dishes containing a monolayer of cortical astroglia in MEM containing $\mathrm{N}_{2}$ supplements (Bottenstein and Sato, 1979), sodium pyruvate $(1 \mathrm{~mm})$ (Sigma, St. Louis, $\mathrm{MO}$ ), and ovalbumin $(0.1 \%$ ) (Sigma). After $3 \mathrm{~d}$, cytosine arabinoside (5 $\mu \mathrm{M})$ (Sigma) was added to inhibit proliferation of glial cells. Neurons were maintained for up to 5 weeks by exchanging one third of the media once a week.

Immunocytochemistry. Cell cultures were fixed at 4, 11, and $18 \mathrm{~d}$ in vitro with $4 \%$ paraformaldehyde in PBS, $\mathrm{pH} 7.2$, containing $0.12 \mathrm{M}$ sucrose, for $30 \mathrm{~min}$ at $37^{\circ} \mathrm{C}$. Cells were permeabilized with $0.25 \%$ Triton X-100 for 5 min, and nonspecific binding sites were blocked with a solution of $10 \%$ bovine serum albumin (BSA) in PBS. Cells were simultaneously incubated in two primary antibodies diluted in $1 \%$ BSA in PBS at $4^{\circ} \mathrm{C}$ overnight: anti-GAD-6, a mouse monoclonal antibody that recognizes the $65 \mathrm{kDa}$ form of GAD [Developmental Studies Hybridoma Bank, Iowa City, IA (Chang and Gottlieb, 1988)], and anti-synaptophysin, an affinitypurified rabbit polyclonal (Navone et al., 1986) (kindly provided by P. De Camilli, Yale University Medical School) (diluted 1:8000). Antibody binding was visualized by incubating cells with a biotinylated anti-mouse secondary antibody, followed by fluorescein-labeled streptavidin (both from Vector, Burlingame, CA) (diluted 1:500), and an anti-rabbit rhodamine-labeled secondary antibody (Boehringer Mannheim Biochemicals, Indianapolis, IN) (diluted 1:200), a combination that yielded the highest signal-to-noise ratio for both antibodies. For analysis of dendritic arbors, some sister cultures were incubated with an antibody against MAP2 (AP14; Binder et al., 1986) (kindly provided by E. Torre, University of Virginia Medical School). For all of the immunocytochemical procedures, control cultures were incubated with $2 \%$ nonimmune serum or $1 \%$ BSA in place of primary antibody. In addition, when two antibodies were used simultaneously, staining was compared with that obtained in cultures incubated with anti-GAD65 or anti-synaptophysin alone.

Tetrodotoxin (TTX) exposure. Cultured hippocampal neurons were exposed continuously to the sodium channel blocker TTX (Sigma) $(1 \mu \mathrm{M})$ for $15 \mathrm{~d}$, beginning from day 3 (just before the formation of synapses). When renewed every $3 \mathrm{~d}$, this concentration of TTX has proven effective in eliminating action potentials during prolonged periods in neurons cultured according to the protocol used in the present study (Van Huizen et al., 1985; Ramakers et al., 1993; Craig et al., 1994; Verderio et al., 1994; D. Benson, unpublished observations).

Light microscopy. Immunostaining was visualized using a Zeiss Axiophot microscope equipped for the visualization of fluorescence and integrated with an image analysis system (NeuroZoom, Scripps Research Institute, La Jolla, CA, and Mount Sinai School of Medicine, New York, NY). Neurons were visualized through a $40 \times$ or $63 \times$ objective, and images were captured using an Optronix CCD camera and transferred to either a DEC or Macintosh computer workstation. The localization of presynaptic specializations labeled by antibodies against synaptophysin or

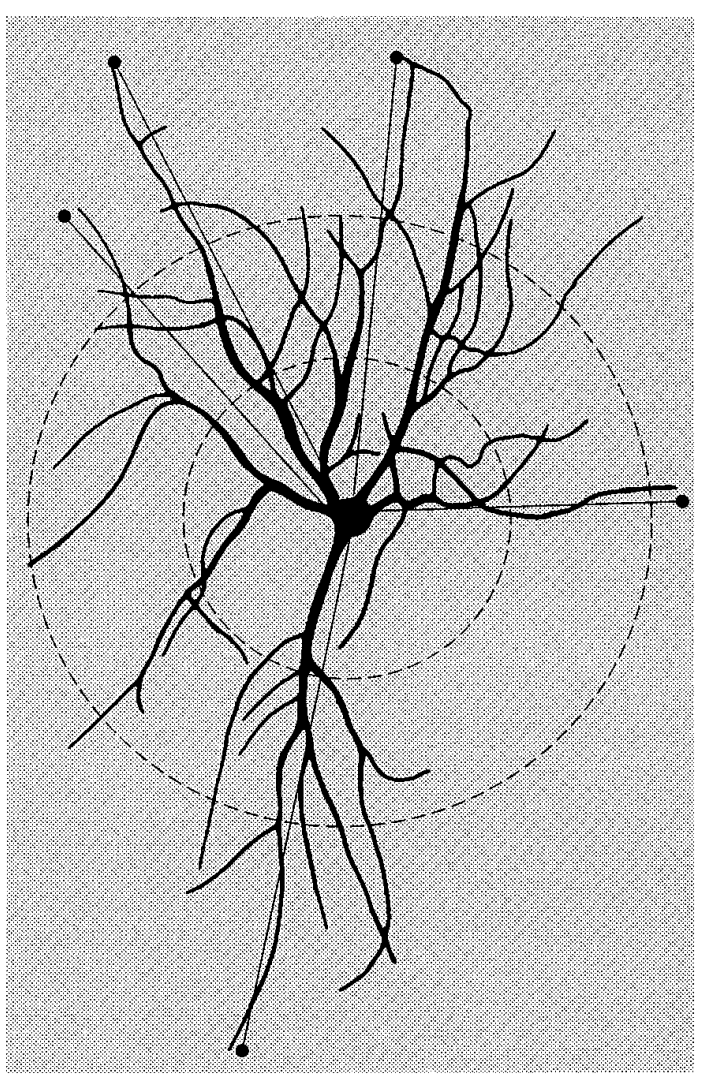

Figure 1. Diagram of a neuron soma and dendrites illustrating modified Scholl analysis. For each of the five primary dendrites in the neuron illustrated, a ray measures the distance from the soma to the farthest extent of the dendritic branches. The rays are averaged and divided by 3 , and the resultant length $(x)$ is used for the radius of the innermost circle. For the outer circle, $r=2 x$. In this way the neuron is divided into four regions: soma, proximal, middle, and distal. See Materials and Methods for details.

GAD65 were mapped directly onto the image, and positions were stored as coordinates. Most neuronal processes in culture were thin enough to be visualized in a single optical plane and could therefore be captured in a single frame, but the relative thickness of the cell soma frequently necessitated the collection of images from several focal planes. In this way the total number of presynaptic terminals and the percentage of terminals containing GAD65 were determined. To ensure that we were counting all labeled terminals, we carried out pilot studies using a confocal laser scanning microscope (Zeiss LSM410). Although focus was sharper with the confocal microscope, the same number of labeled terminals was clearly visible when a conventional microscope was used.

A modified Scholl analysis (Scholl, 1953) was used to compare quantitatively the relative positions of non-GABAergic and GABAergic presynaptic terminals. A line was traced around the cell soma, and two concentric circles of increasing radius were drawn around it. Because neurons of increasing age were also increasing in size, the radii of the concentric circles were adjusted systematically such that the dendritic tree was divided into three roughly equal regions: proximal, middle, and distal. The distance between the circles was determined by measuring rays emanating from the edge of the soma to the most distal terminal branch for each primary dendrite, taking the average length, and dividing by 3 (Fig. 1). The percentage of total dendritic length contained within proximal regions averaged 34\% ( \pm 2$)$, within the middle regions, $29 \%( \pm 2)$, and within the distal regions, $37 \%( \pm 2)$. The number of synapses that fell within each region was counted. Dendritic arbors were mapped using synaptophysin staining and phase-contrast microscopy or sister cultures that were immunostained for MAP2 (Benson et al., 1994). For each neuron, we determined the number of primary branches arising from the cell soma, the total length and number of dendritic segments, and the number of terminal branches. At least 10 cells per experiment and at least 

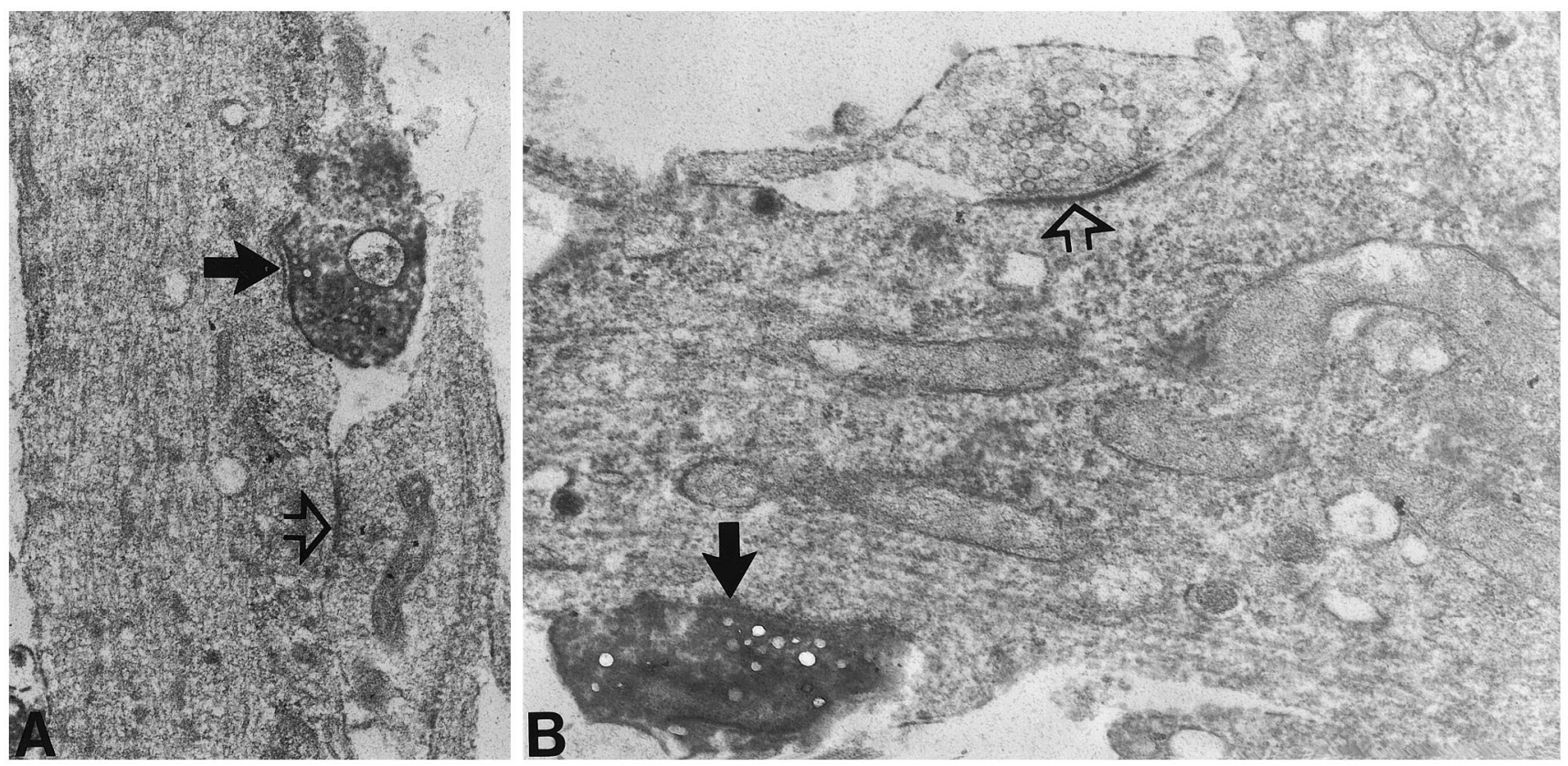

Figure 2. GAD65 labels synaptic boutons. Electron photomicrographs showing GAD65 immunolabeling of synaptic terminals in 18-d-old cultured hippocampal neurons. In both $A$ and $B, \mathrm{DAB}$ reaction product is localized to vesicle-filled synaptic boutons contacting unlabeled dendrites (filled arrows). Open arrows indicate unlabeled axo-dendritic synaptic terminals. Magnification: $20,000 \times(A) ; 30,400 \times(B)$.

30 cells per age were examined in this manner, unless noted otherwise. Significant differences were detected by ANOVA, and the sources of differences were detected using Scheffé's F procedure for post hoc comparisons.

Electron microscopy. To assess GAD and GABA immunolocalization, we used a method modified from that of Caserta and Barker (1994). Eleven- and 18-d-old neural cultures were fixed in a $2 \%$ paraformaldehyde $/ 0.12 \mathrm{M}$ sucrose $/ 0.1 \%$ glutaraldehyde/PBS solution. Cells were then treated with $0.5 \mathrm{mg} / \mathrm{ml}$ sodium borohydride, permeabilized with $0.02 \%$ saponin in PBS, and blocked with $10 \%$ BSA in PBS. Cells were incubated overnight at $4^{\circ} \mathrm{C}$ in undiluted anti-GAD65 supernatant. Labeling was detected using the Vector "Elite" avidin-biotin kit with diaminobenzidine as chromogen. The cells were postfixed in $1 \%$ glutaraldehyde and washed in PBS. The cells were then treated with $2 \%$ osmium tetroxide, dehydrated through graded ethanols, and embedded using propylene oxide and epon. Once the epon polymerized the cells, they were removed from the cover glass using liquid nitrogen. Ultrathin sections were cut and mounted on copper grids and then stained by uranyl acetate and lead citrate.

\section{RESULTS}

\section{Normal maturational sequence of cultured hippocampal neurons}

Neurons that were cultured for 4, 11, and $18 \mathrm{~d}$ were used for most of the experiments described in this study. Each of these ages represents a different developmental stage with regard to the formation of synapses (Fletcher et al., 1991). At 4 d, axons have emerged from cell somata, dendrites are beginning to form, and synaptogenesis has just begun. At $11 \mathrm{~d}$, synapses are forming rapidly and dendrites are significantly more mature, but dendritic spines have not yet formed. By $18 \mathrm{~d}$ in culture, neurons have well developed axonal and dendritic arbors, and pyramidal-type neurons have elaborated dendritic spines.

\section{Identification of GABAergic and non-GABAergic terminals}

To identify GABAergic and non-GABAergic synaptic terminals, cultures were immunostained simultaneously with antibodies against GAD65 and synaptophysin (Fletcher et al., 1991; Benson et al., 1994). Synaptophysin immunolabeling identified the total number of presynaptic terminals, whereas GAD65 identified those that were also GABAergic. By light microscopy, GAD65containing boutons apposed to dendrites were in precise register with synaptophysin-labeled boutons. Electron microscopy confirmed that in both 11- and 18-d-old cultures, GAD65-labeled boutons apposing dendritic membranes were presynaptic. Labeled axon terminals that contained clusters of vesicles were apposed to a slightly thickened postsynaptic density (Fig. 2). GAD65-labeled synaptic boutons were never observed to appose dendritic spines.

At $4 \mathrm{~d}$ in culture, the light microscopic localization of both GAD65 and synaptophysin immunolabeling was most prominent in a region of cytoplasm adjacent to the nucleus that is most likely to be the Golgi apparatus. GAD65 label also extended diffusely into the surrounding cytoplasm, but was rarely observed in brightly stained granules that might be indicative of vesicles (Fig. $3 A$ ). By contrast, synaptophysin label was observed in granules that have been demonstrated to be vesicles by electron microscopy (Fletcher et al., 1991). Labeled vesicles extended into axons and clustered infrequently in presynaptic terminal boutons (Fig. $3 B$ ). By $7 \mathrm{~d}$ in culture, GAD65 and synaptophysin immunolabeling were prominent in bouton-like structures, and synaptophysin was no longer prominent in the soma (not shown). At 11 and $18 \mathrm{~d}$ in culture, presynaptic terminals were clearly labeled with both GAD65 and synaptophysin staining (Fig. $3 D-E$ ). These data closely concur with what has been reported previously (Fletcher et al., 1991; Benson et al., 1994; Solimena et al., 1994).

\section{Rate of formation of synapses}

At $4 \mathrm{~d}$ in culture, there was an average of 22 synaptophysinlabeled boutons on the soma and dendrites of neurons receiving synaptic input, but many neurons had none. The number of synaptophysin-labeled terminals did not correlate with somal size, which was approximated by area. The number of boutons per 

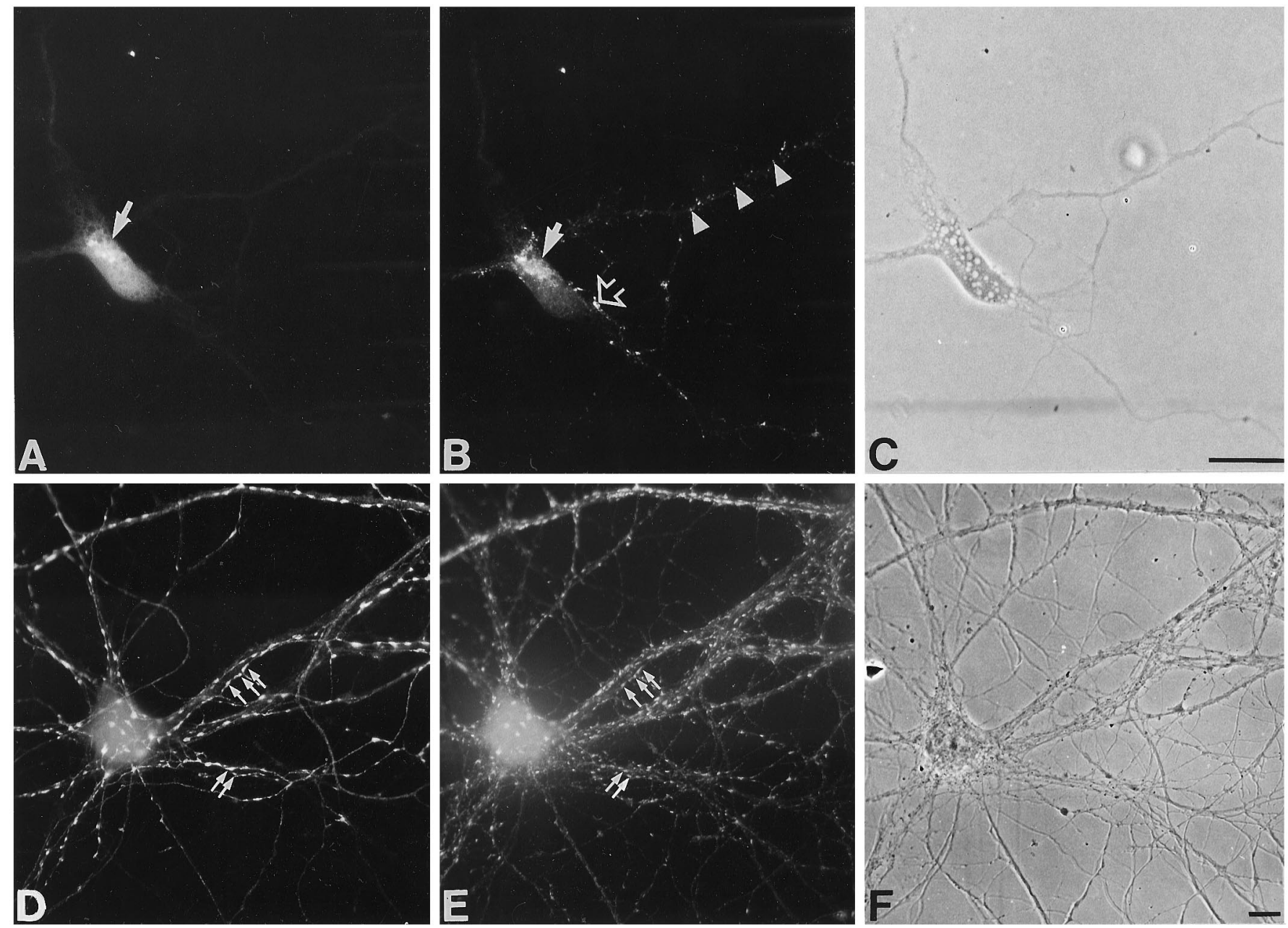

Figure 3. Fluorescence $(A, B, D, E)$ and phase-contrast $(C, F)$ photomicrographs showing the distribution of GAD65 $(A, D)$ and synaptophysin $(B, E)$ immunoreactivity in cultured hippocampal neurons. In 4-d-old neurons (A-C), GAD65 immunoreactivity is restricted to cell somata with a slightly greater concentration in what is probably the Golgi apparatus $(A$, arrow). Synaptophysin immunoreactivity is concentrated in the same area of the soma $(B$, arrow), but vesicle-like particles are also distributed in growing neurites (arrowheads) and occasional presynaptic clusters are observed (open arrowhead). At $18 \mathrm{~d}$ in culture $(D-F)$, GAD65 immunoreactivity is found throughout axons and brightly labeled presynaptic boutons (small arrows), the latter of which correspond precisely to a subpopulation of the synaptophysin-labeled boutons shown in $E$ (small arrows). Virtually all of the synaptophysin label in $E$ is localized to brightly labeled terminals that have been demonstrated to be synaptic boutons (see introductory remarks). Scale bars, $10 \mu \mathrm{m}$.

micrometer increased linearly over time and reached an average of $6.1 / 10 \mu \mathrm{m}$ at $18 \mathrm{~d}$ (Fig. $4 A$ ). The average rate of synaptophysinlabeled bouton development was similar to that of the overall increase in dendritic length, although the rate of dendritic outgrowth exceeded that of synapse development between 11 and $18 \mathrm{~d}$ in culture ( $p<0.04$; Fig. 4). Dendritic length increased more by addition of new branches than by lengthening of existing branches.

The development of GAD65-labeled boutons followed that of synaptophysin. At $4 \mathrm{~d}$ in culture, $>93 \%$ of the neurons were not contacted by GAD65-labeled boutons, but two of the neurons examined had two or three GAD65-positive boutons. At $11 \mathrm{~d}$ in culture, $\sim 68 \%$ of the neurons were contacted by GAD65-labeled boutons, with an average of 1.6 boutons $/ 10 \mu \mathrm{m}$. At $18 \mathrm{~d}$ in culture, all but $15 \%$ of the neurons were contacted by GAD65 boutons, and there was an average of 1.8 boutons $/ 10 \mu \mathrm{m}$; however, the neurons lacking GAD65 boutons do not seem to be incapable of receiving such inputs, because by 4 weeks in culture, all neurons were contacted by GAD65-labeled boutons. Although GAD65labeled terminals were found throughout the somatodendritic domain, they appeared more clustered around cell somata.

\section{Segregation of terminals to cellular domains}

To determine how GAD65-positive and -negative synaptic terminals were distributed along the somatodendritic domain, we mapped the location of GAD65- and synaptophysin-containing terminals using computer software that would assign each identified bouton an $x, y, z$ coordinate. The resulting synaptic terminal maps were divided into four areas: the first was the cell soma, and the latter three-proximal, middle, and distal-represented relatively equal divisions of the dendritic tree (see Materials and Methods).

At $4 \mathrm{~d}$ in culture, there were extremely few GABAergic terminals on any neuron, and these were mostly on dendrites. In addition, synaptophysin-immunoreactive terminals were evenly distributed across somal, proximal, middle, and distal regions. By $7 \mathrm{~d}$ in culture, the numbers of both GABAergic and nonGABAergic terminals had increased over all regions of their target neurons. In comparing the distribution of GABAergic and non-GABAergic terminals, there was a trend toward having a greater percentage of GABAergic terminals on cell somata $(38 \%)$ than on dendrites $(18 \%)$, although the difference was not statis- 
A.
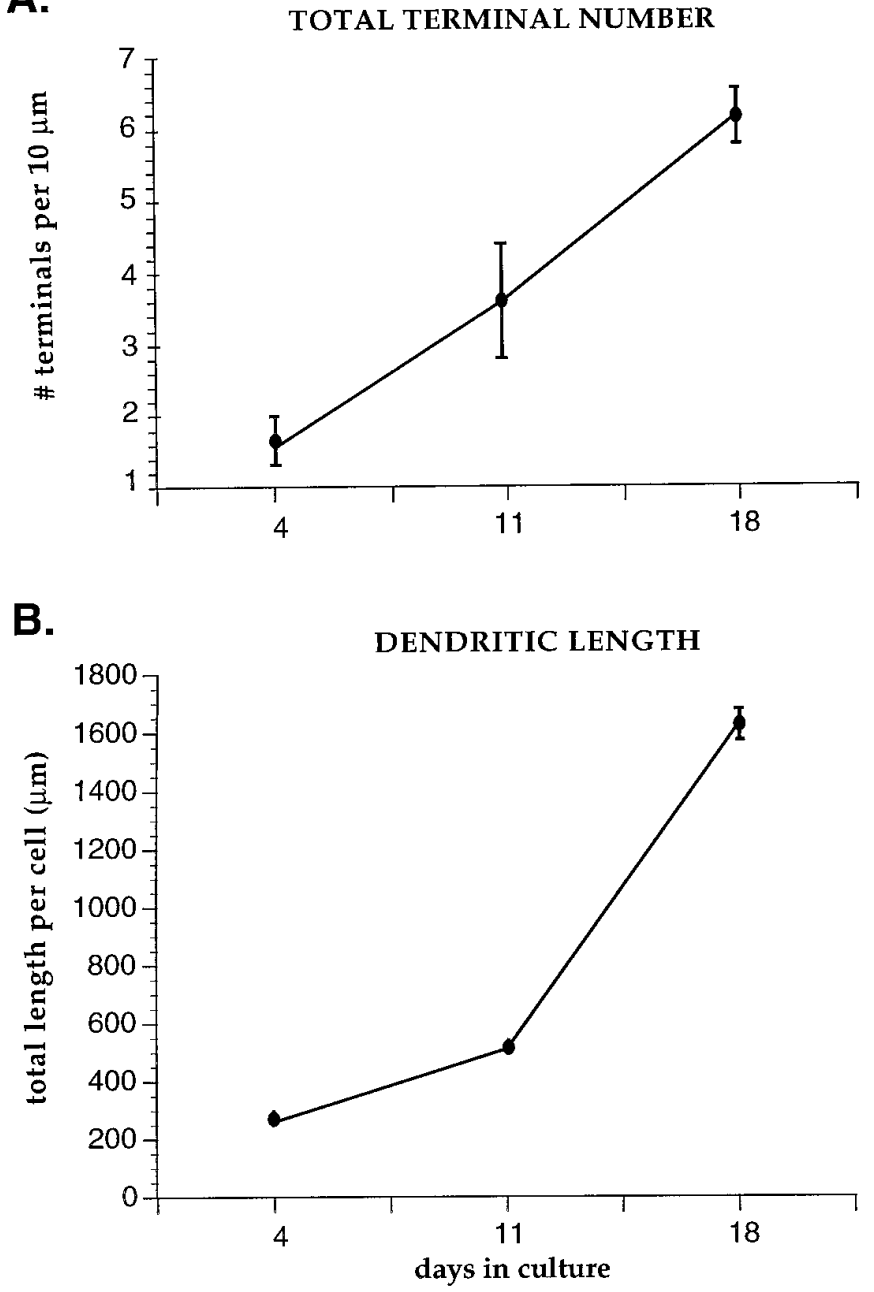

Figure 4. Graphs showing increase in synaptic terminal number $(A)$ and dendritic length $(B)$ during development in culture. Synaptic terminal number $(A)$ was determined by counting the total number of synaptophysin-labeled boutons on individual neurons grown in culture. Dendritic length was determined by measuring all dendritic branches of MAP2-labeled neurons. $n=30$ except for 18 -d-old cells in $A$, where $n=$ 20. Error bars represent SEM.

tically significant $(n=10, p<0.3)$. By $11 \mathrm{~d}$ in culture, the percentages of synaptic terminals on cell somata that were GABAergic or non-GABAergic were approximately equal (51 vs $49 \%$ ), whereas in all dendritic regions, the percentage of terminals that were GABAergic lagged significantly behind that which were nonGABAergic (Fig. $5 A$ ). By $18 \mathrm{~d}$ in culture, the percentage of synaptic terminals that were GABAergic had increased on cell somata such that it exceeded the percentage of non-GABAergic somatic terminals by $33 \%$. On all dendritic regions, the percentage of synaptic terminals that were GABAergic was less than half that which were non-GABAergic (Fig. 5B). The percentage of total synaptic terminals that were GABAergic decreased sharply over a proximal-to-distal gradient whereas the percentage that was non-GABAergic increased.

At $18 \mathrm{~d}$ in culture, of the total number of GABAergic terminals, $12 \%$ were on somata and $88 \%$ were on dendrites. In contrast, $4 \%$ of non-GABAergic terminals were on somata, and $96 \%$ were on dendrites ( $p<0.0001$ by $\chi^{2}$ analysis).

Comparison of the rates of GAD65-positive and GAD65-

\section{DISTRIBUTION OF GABAergic TERMINALS}
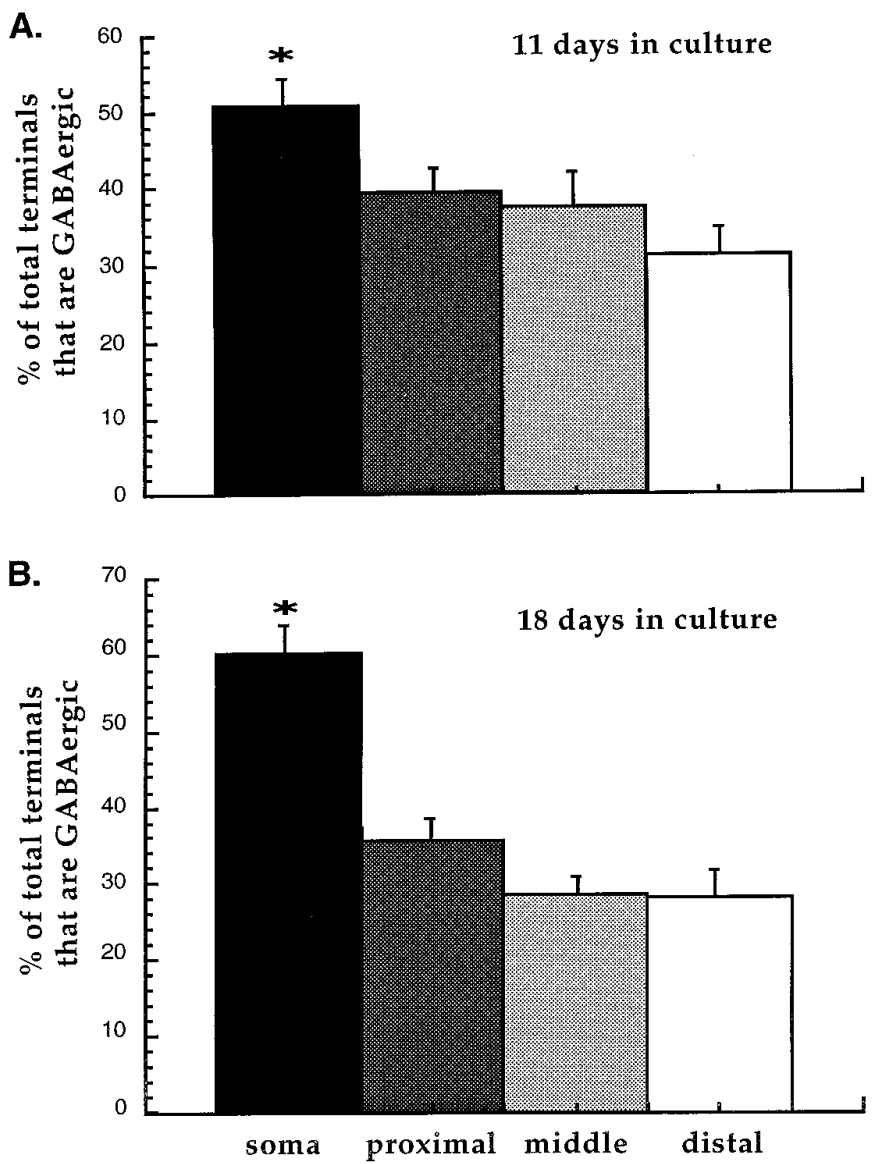

Figure 5. Percentage and distribution of terminals that were GABAergic at 11 and $18 \mathrm{~d}$ in culture. Bar graph shows the percentage of total (synaptophysin-immunoreactive) terminals that contained GAD65 immunoreactivity within soma, proximal, middle, and distal regions (as defined in Materials and Methods). At $11 \mathrm{~d}$, the percentage of terminals that was GABAergic is significantly greater on somata than elsewhere on the neuron. By $18 \mathrm{~d}$ in culture, an even greater proportion of somatic terminals contained GAD65 label. Asterisks indicate columns that are significantly different from all other columns $(A, p<0.02 ; B, p<0.001)$. Error bars represent SEM.

negative terminal innervation of somata revealed that the number of GAD65-positive terminals increased more rapidly than that of GAD65-negative terminals (Fig. 6A). A similar comparison restricted to dendritic trees (that is, excluding cell somata) showed that GAD65-positive terminals formed at a much slower rate than did GAD65-negative terminals (Fig. 6B).

We next examined the distribution of somatic boutons on relatively old cultures ( $31 \mathrm{~d}$ in vitro) to assess the stability of the enhanced GABAergic innervation on somata. The proportion of somatic GABAergic synapses did not change significantly over this time.

\section{Do GABAergic terminals affect the formation of non-GABAergic terminals?}

We compared the total number of synaptophysin-labeled terminals on the somata of neurons receiving GABAergic terminals with those that were devoid of GABAergic terminals. The total number of synaptophysin-labeled terminals did not vary between these two groups [2.54 boutons/10 $\mu \mathrm{m}^{2} \pm 0.19$ and 2.20 bou- 


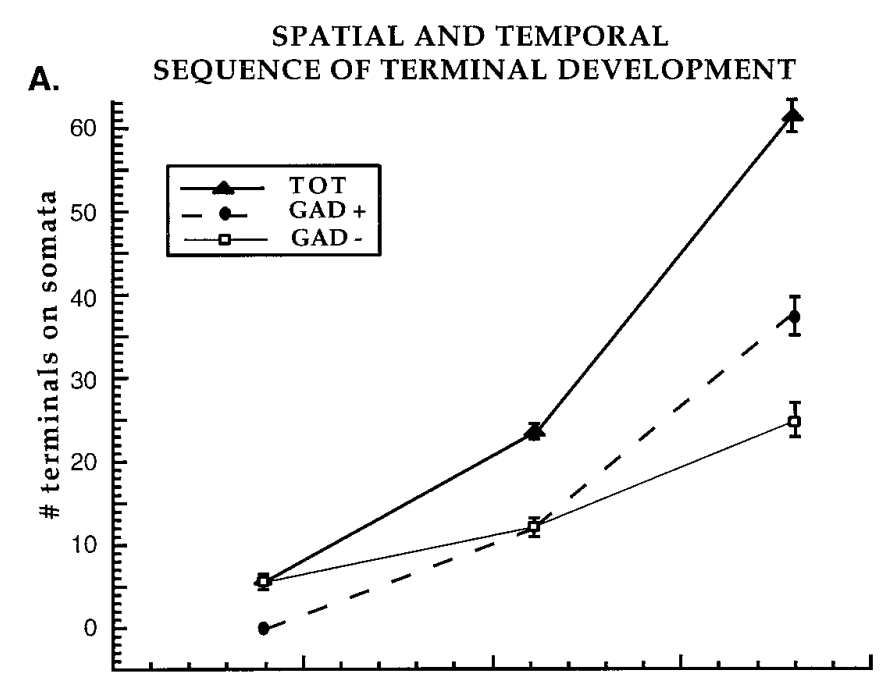

B.

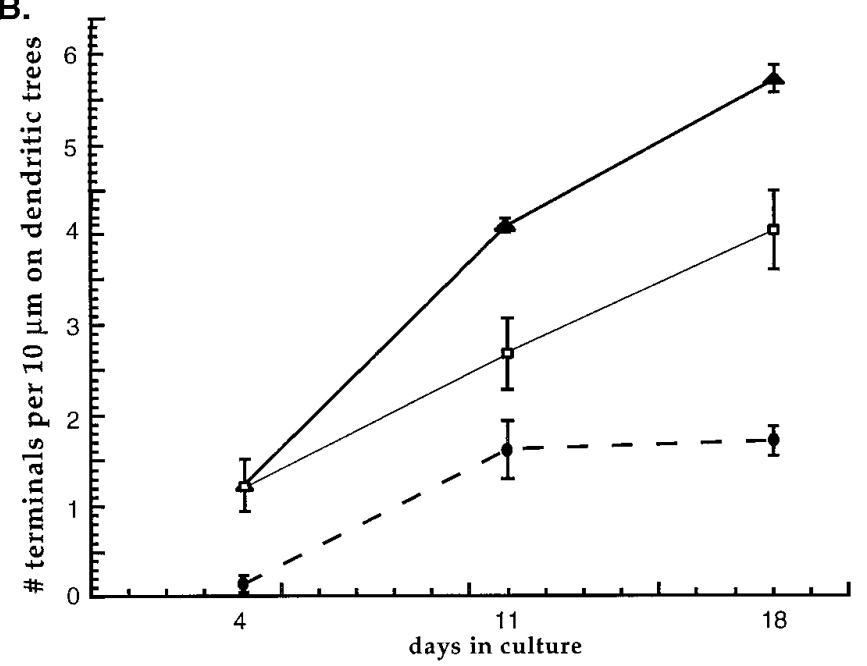

Figure 6. Rates of synaptic terminal development on somata $(A)$ and dendritic trees $(B)$. On somata $(A)$, the rate at which GAD-positive terminals form ( $G A D+$; dashed line $)$ increases more rapidly than the rate for GAD-negative terminals $(G A D-;$ solid thin line). The rate at which all terminals develop is indicated by the solid thick line. Differences in slopes $(\mathrm{GAD}+\mathrm{vs} \mathrm{GAD}-)$ are statistically significant by paired $t$ test: between 4 and $11 \mathrm{~d}, p<0.002$, and between 11 and $18 \mathrm{~d}, p<0.003$. On dendrites $(B)$, the rate at which GAD + terminals form flattens beyond $11 \mathrm{~d}$ in culture, whereas GAD - terminals continue to proliferate at a steady rate $(p<$ 0.0002).

tons $/ 10 \mu \mathrm{m}^{2} \pm 0.26$, respectively $\left.(n=20)\right]$. The presence and extent of GAD innervation of somata correlated precisely with a concomitant reduction in the number of GAD-negative terminals in somata.

\section{Effects of activity blockade on the segregation of synapses}

Neuronal activity was chronically blocked by exposing neuron cultures to $1 \mu \mathrm{M}$ TTX from the third day after plating. TTX was renewed every $3 \mathrm{~d}$, a frequency that has been demonstrated to eliminate evoked potentials (Craig et al., 1994; Verderio et al., 1994). Even after a 15 d exposure, neurons appeared healthy and qualitatively indistinguishable from untreated neurons. By labeling cultures with antibodies against GABA, which more completely labels cell somata than GAD65 does, we determined that $6 \%$ of the neurons in TTX-treated cultures were GABAergic.
This percentage did not differ significantly from that in sister cultures or from untreated cultures analyzed previously (Benson et al., 1994). The effect of TTX on dendritic outgrowth and maturation was assessed by counting the number of primary and terminal dendritic branches and by measuring individual branch lengths in cultures immunocytochemically labeled with MAP2. Prolonged treatment with TTX reduced the overall extent of dendritic arbors primarily by decreasing the numbers of terminal branches and the lengths of primary dendrites (Table 1). By contrast, TTX treatment had no effect on the distribution or proportion of GAD65-labeled terminals on cell somata or on proximal, middle, and distal dendrites, nor did TTX treatment affect the number of synaptophysin boutons per micron (Table 1).

\section{DISCUSSION}

In the present study, we used cultured hippocampal neurons to examine the spatial and temporal sequence leading to the proper sorting of excitatory and inhibitory synaptic terminals to different cellular domains, and the role of neural activity in this process. There were three major findings. (1) From the time at which they began to form, GABAergic synaptic terminals developed more rapidly and eventually outnumbered non-GABAergic terminals on neuronal somata, whereas non-GABAergic terminals predominated on dendrites. (2) In the absence of GABAergic synaptic terminals, non-GABAergic terminals were not inhibited from forming synapses on cell somata. (3) These initial stages of synaptic terminal segregation occurred normally in the absence of neural activity, although dendritic arborization was inhibited. Collectively, these data rule out the hypothesis that GABAergic and non-GABAergic terminals initially are distributed randomly and only through a later process rearrange to their adult-like pattern of relatively restricted distribution. Rather, the data support a model whereby from the earliest stages of GABAergic terminal formation, inhibitory synaptic terminals are maintained preferentially on cell somata. Interestingly, however, this does not imply that non-GABAergic terminals are inhibited from forming contacts with cell somata, because they readily achieved a normal total synaptic density on somata lacking GAD terminals. Rather, it may be the case that GABAergic terminals inhibit or repulse non-GABAergic ones, or they compete more successfully for some attractant molecule or trophic factor.

\section{Development of GAD-containing terminals and synaptic inhibition}

The appearance of GAD-labeled boutons in the present study correlates closely with the reported appearance of functional synaptic inhibition in cultured hippocampal neurons (Basarsky et al., 1994) as well as in hippocampus of approximately the same age in vivo (Zhang et al., 1991). $\mathrm{GABA}_{\mathrm{A}}$ receptors are present even before GAD-labeled boutons (Killisch et al., 1991). Therefore, at least some GAD-labeled boutons are likely to provide inhibition at the earliest stages examined, although it is possible that neurotransmitter release from some of the GAD-labeled boutons results in depolarizing potentials (Ben-Ari et al., 1989; Fiszman et al., 1990; Xie and Smart, 1991). The fact that the numbers of GAD-labeled boutons continue to increase over the entire neuron suggests that such boutons are not transient and will eventually, if not immediately, contribute to synaptic inhibition.

\section{Development of non-GABAergic terminals}

Hippocampal neurons, when cultured as described, are composed only of interneurons and pyramidal neurons (Goslin and Banker, 1991). The vast majority of interneurons are GABAergic, and 


\section{Control}

$\begin{aligned} 61.3 & \pm 9.8(\mathrm{n}=15) \\ 6.4 & \pm 0.8(\mathrm{n}=15) \\ 1521 & \pm 110.6(\mathrm{n}=32) \\ 7.1 & \pm 0.5(\mathrm{n}=32) \\ 17 & \pm 1.2(\mathrm{n}=32) \\ 79 & \pm 6.0(\mathrm{n}=32)\end{aligned}$

TTX

$\%$ boutons on soma that contain GAD65
Total boutons/10 $\mu \mathrm{m}$
Total dendritic length $(\mu \mathrm{m})$
Number of primary dendrites
Number of terminal branches
Length primary branches $(\mu \mathrm{m})$

$\%$ boutons on soma that contain GAD65

Number of prim

Length primary branches $(\mu \mathrm{m})$

$$
\begin{aligned}
62.0 & \pm 10.9(\mathrm{n}=15) \\
6.5 & \pm 0.6(\mathrm{n}=15) \\
1101.3 & \pm 110.7^{*}(\mathrm{n}=30) \\
6.1 & \pm 2.8(\mathrm{n}=30) \\
11.9 & \pm 1.4^{*}(\mathrm{n}=30) \\
37 & \pm 3.9^{* *}(\mathrm{n}=30)
\end{aligned}
$$

${ }^{*} p<0.01 ;{ }^{* *} p<0.001$.

their synaptic terminals will contain GAD (Benson et al., 1994). Because pyramidal neurons are glutamatergic (Streit, 1984), terminals lacking GAD are probably excitatory.

At all ages, most terminal boutons on cultured hippocampal neurons lacked GAD. This is not surprising, because pyramidal neurons greatly outnumber interneurons (16:1) in these cultures (Benson et al., 1994). Most of the non-GABAergic boutons terminate on dendrites, an observation that parallels the distribution of asymmetric synapses in the adult hippocampus in vivo (Andersen et al., 1966). The rate at which non-GABAergic terminals form on dendrites increases more rapidly than that of GABAergic terminals between 11 and $18 \mathrm{~d}$ in culture, a period coinciding with the development of dendritic spines (Banker and Waxman, 1988; Papa et al., 1995; Benson et al., 1996). Dendritic spines are the principal sites for excitatory synaptic terminals (Horner, 1993; Harris and Kater, 1994) and have extremely few GABAergic terminals in vivo and in vitro (Seress and Ribak, 1990; Craig et al., 1993; present study). It is not known how dendritic spines are formed, but an appealing hypothesis is that excitatory synaptic terminal proliferation may serve to induce incipient dendritic spines.

\section{Segregation of GABAergic and non-GABAergic terminals}

How do GABAergic and non-GABAergic terminals become selectively distributed on their targets? The present data show that the rate of formation of GABAergic terminals exceeds that of non-GABAergic terminals on somata, but it lags behind nonGABAergic terminals on dendrites. This supports the notion that attractive and/or repulsive forces may be used for localized synaptic terminal targeting on cellular domains, much like what has been described for guiding axons to more distant targets (Goodman, 1996). One possibility is that the greater number of somatic GABAergic terminals may arise by localized repulsion of nonGABAergic terminals from somata; however, our data suggest that this is unlikely. In the absence of GABAergic input, nonGABAergic terminals achieve the same total synaptic terminal density on somata observed in the presence of GABAergic input. Rather, cell somata seem to provide a particularly permissive environment for the formation of GABAergic terminals. Conditions that favor GABAergic innervation could be generated by a number of mechanisms. GABAergic axons might express a unique receptor corresponding to a guidance molecule that has an enhanced somatic distribution; GABAergic axons may compete more effectively for a soma-localized trophic factor; or GABAergic axons may inhibit or repel non-GABAergic axons.

On dendrites, non-GABAergic terminals form more rapidly than GABAergic terminals. They may respond to an attractive molecule(s) emanating from dendrites, but given that nonGABAergic neurons greatly outnumber GABAergic neurons, an attractive force would not be necessary to achieve a greater density of synaptic terminals. Likewise, because GABAergic axons are capable of forming distally located synapses, it is unlikely that they are selectively repulsed from dendrites, or at least from dendritic shafts; however, they may be repulsed from dendritic spines. We found no GABAergic synaptic terminals on dendritic spines, and GAD-immunoreactive terminals do not seem to colocalize with the dendritic spine-localized glutamate receptor subunit GluR1 in cultured hippocampal neurons (Craig et al., 1994).

\section{Comparative aspects of synaptic terminal distribution in vivo and in vitro}

The preference that GABAergic terminals show for somata and that non-GABAergic terminals show for dendrites is a pattern that resembles the distribution of GABAergic or symmetric and asymmetric terminals during early postnatal development of hippocampus and neocortex (Blue and Parnavelas, 1983; Bähr and Wolff, 1985; Seress et al., 1989). In adult rat hippocampus, however, nearly all somatic synapses are symmetric and presumably inhibitory (Gottlieb and Cowan, 1972), a state never attained by GAD-labeled terminals in the cultured neurons examined in the present study. It is possible that if neurons could be grown in culture indefinitely, they might eventually display an adult-like synaptic distribution pattern either by synaptic remodeling or elimination or by previously unlabeled terminals becoming immunoreactive for GAD. If this were the case, we would predict the proportion of GABAergic terminals on neuronal somata to increase over time in culture, but we found the proportion to be relatively stable after $18 \mathrm{~d}$ in culture. Furthermore, it is unlikely that unlabeled terminals would become GABAergic, because there was no evidence of either a change in the total number of neurons expressing GAD or GABA or in the intensity of GAD labeling. It is more likely that the final exclusion of nonGABAergic synapses from cell somata requires the presence of an interaction not available in culture.

The observations in the present study support a two-step model for the segregation of excitatory and inhibitory synapses on postsynaptic neurons. In the first step, excitatory and inhibitory synapses are sorted differentially to separate cellular target domains, but some overlap remains on cell somata. In the second step, remaining excitatory terminals are eliminated from cell somata, but the original distribution pattern is maintained on dendrites. Such a two-step process is similar to what has been described for the regional restriction of synapses in several areas of vertebrate brain where weaker and/or inappropriate connections are eliminated and stronger and/or appropriate connections are maintained (Debski et al., 1990; Goodman and Shatz, 1993; O'Leary and Koester, 1993). It follows that the observations made in the present study apply to the early acquisition of synapses rather than to their final organization. 


\section{Terminal segregation and neuronal activity}

A number of studies have demonstrated that neurotransmitters can directly affect growth cone movement (Haydon et al., 1984; Michler-Stuke and Wolff, 1987; McCobb et al., 1988), and given the putative neurotrophic role for GABA (Hansen et al., 1984; Taylor et al., 1990), GABA release is an obvious candidate for initiating preferential interactions with cell somata or for repelling non-GABAergic growth cones. However, unlike the activitydependent pruning of synaptic terminals that is often observed in vertebrates (Goodman and Shatz, 1993), the present data demonstrate that segregation of excitatory and inhibitory terminals occurs normally in the absence of activity-evoked neurotransmitter release. Thus, if GABA serves as the signaling molecule, small amounts released spontaneously must be sufficient to engage somatic GABA receptors.

In cultured rat cerebral cortex, long-term exposure to TTX depletes GABA (Ramakers et al., 1994). Thus, it is possible that GABA levels were reduced in our cultures; however, there were no overt decreases in the intensity of GAD immunofluorescence, in the number of GABA-immunoreactive neurons, or in the number of GAD-containing boutons after TTX treatment. The apparent absence of any change in GAD immunocytochemistry concurs with what has been observed in the cerebral cortex in vivo. After monocular deprivation in adult monkeys, levels of immunocytochemically localized GABA and GAD as well as GAD67 mRNA are decreased in the eye-dominance columns of primary visual cortex corresponding to the deprived eye relative to the normal eye columns (Hendry and Jones, 1986; Benson et al., 1991); however, the monocular crescent representation in visual cortex that receives information solely from the contralateral, deprived eye shows no change in GABA or GAD immunoreactivity (Hendry and Carder, 1992). Changes in the levels of GABA and GAD seem to be restricted to regions where an imbalance in the levels of activity arising from the two eyes occurs. In the present study, all synaptic interactions are affected equally by TTX, and thus no imbalance in activity exists. Therefore, GAD levels would not be expected to change, consistent with the present observations.

The present results, however, demonstrated that chronic activity blockade did lead to decreased dendritic outgrowth. A number of studies have suggested that synaptogenesis may be retarded when neural activity is absent (Zanka and Jones, 1982; Van Huizen et al., 1985), but our results suggest instead that a decrease in synapse number may be attributable to a decrease in available synaptic sites.

\section{REFERENCES}

Andersen P, Blackstad TW, Lömo T (1966) Location and identification of excitatory synapses on hippocampal pyramidal cells. Exp Brain Res $1: 236-248$.

Andersen P, Eccles JC, Løyning Y (1964) Pathway of postsynaptic inhibition in the hippocampus. J Neurophysiol 27:608-619.

Bähr S, Wolff JR (1985) Postnatal development of axosomatic synapses in the rat visual cortex: morphogenesis and quantitative evaluation. J Comp Neurol 233:405-420.

Banker GA, Cowan WM (1977) Rat hippocampal neurons in dispersed cell culture. Brain Res 126:397-425.

Banker GA, Waxman AB (1988) Hippocampal neurons generate natural shapes in cell culture. In: Intrinsic determinants of neuronal form and function (Lasek RJ, Black MM, eds), pp 61-82. New York: Alan R. Liss.

Basarsky TA, Parpura V, Haydon PG (1994) Hippocampal synaptogenesis in cell culture: developmental time course of synapse formation, calcium influx, and synaptic protein distribution. J Neurosci 12:6402-6411.
Bastiani MJ, Goodman CS (1986) Guidance of neuronal growth cones in the grasshopper embryo. III. Recognition of specific glial pathways. J Neurosci 6:3542-3551.

Bate CM (1976) Pioneer neurons in an insect embryo. Nature 260:54-56. Beaulieu C, Colonnier M (1985) A laminar analysis of the number of round-asymmetrical and flat-symmetrical synapses on spines, dendritic trunks, and cell bodies in area 17 of the cat. J Comp Neurol 231:180-189.

Ben-Ari Y, Cherubini E, Corradetti R, Gaiarsa JL (1989) Giant synaptic potentials in immature rat CA3 hippocampal neurons. J Physiol (Lond) 416:303-325.

Benson DL, Isackson PJ, Gall CM, Jones EG (1991) Differential effects of monocular deprivation on glutamic acid decarboxylase and type-II calcium-calmodulin dependent protein kinase gene expression in the adult monkey visual cortex. J Neurosci 11:31-47.

Benson DL, Mandell JW, Shaw G, Banker G (1996) Compartmentation of alpha-internexin and neurofilament triplet proteins in cultured hippocampal neurons. J Neurocytol 25:181-196.

Benson DL, Watkins FH, Steward O, Banker G (1994) Characterization of GABAergic neurons in hippocampal cell cultures. J Neurocytol 23:279-295.

Binder LI, Frankfurter A, Rebhun LI (1986) Differential localization of MAP2 and tau in mammalian neurons in situ. Ann NY Acad Sci 466:145-167.

Blackstad TW, Flood PR (1963) Ultrastructure of hippocampal axosomatic synapses. Nature 198:542-543.

Blue ME, Parnavelas JG (1983) The formation and maturation of synapses in the visual cortex of the rat. I. Qualitative analysis. J Neurocytol 12:599-616.

Bottenstein JE, Sato GE (1979) Growth of a rat neuroblastoma cell line in serum-free supplemented medium. Proc Natl Acad Sci USA 76:514-519.

Broadie KS (1994) Synaptogenesis in Drosophila: coupling genetics and electrophysiology. J Physiol (Lond) 88:123-139.

Caserta MT, Barker JL (1994) Development of the GABAergic phenotype in murine spinal cord-dorsal root ganglion cultures. Int $\mathbf{J}$ Dev Neurosci 12:753-765.

Chang Y-C, Gottlieb DI (1988) Characterization of the proteins purified with monoclonal antibodies to glutamic acid decarboxylase. J Neurosci 8:2123-2130.

Craig AM, Blackstone CD, Huganir RL, Banker G (1993) The distribution of glutamate receptors in cultured rat hippocampal neurons: postsynaptic clustering of AMPA-selective subunits. Neuron 10:1055-1068.

Craig AM, Blackstone CD, Huganir RL, Banker G (1994) Selective clustering of glutamate and $\gamma$-aminobutyric acid receptors opposite terminals releasing the corresponding neurotransmitters. Proc Natl Acad Sci USA 91:12373-12377.

Debski EA, Cline HT, Constantine-Paton M (1990) Activity-dependent tuning and the NMDA receptor. J Neurobiol 21:18-32.

Fiszman M, Novotny EA, Lange GD, Barker JL (1990) Embryonic and early postnatal hippocampal cells respond to nanomolar concentrations of muscimol. Dev Brain Res 53:186-193.

Fletcher TL, Cameron P, De Camilli P, Banker G (1991) The distribution of synapsin I and synaptophysin in hippocampal neurons developing in culture. J Neurosci 11:1617-1626.

Fox K, Schlagger BL, Glazewski S, O’Leary DDM (1996) Glutamate receptor blockade at cortical synapses disrupts development of thalamocortical and columnar organization in somatosensory cortex. Proc Natl Acad Sci USA 93:5584-5589.

Glanzman DL, Kandel ER, Schacher S (1991) Target-dependent morphological segregation of Aplysia sensory outgrowth in vitro. Neuron 7:903-913.

Goodman CS (1994) The likeness of being: phylogenetically conserved molecular mechanisms of growth cone guidance. Cell 78:353-356.

Goodman CS (1996) Mechanisms and molecules that control growth cone guidance. Annu Rev Neurosci 19:341-377.

Goodman CS, Shatz CJ (1993) Developmental mechanisms that generate precise patterns of neuronal connectivity. Cell [Suppl] 72:77-98.

Goslin K, Banker G (1991) Rat hippocampal neurons in low density culture. In: Culturing nerve cells (Banker G, Goslin K, eds), pp 251-282. Cambridge: MIT.

Gottlieb DI, Cowan WM (1972) On the distribution of axonal terminals containing spheroidal and flattened synaptic vesicles in the hippocampus and dentate gyrus of the rat and cat. Z Zellforsch 129:413-429. 
Gray EG (1959) Axo-somatic and axo-dendritic synapses of the cerebral cortex: an electron microscope study. J Anat 93:420-433.

Hall ZW, Sanes JR (1993) Synaptic structure and development: the neuromuscular junction. Cell [Suppl] 72:99-121.

Hamlyn LH (1963) An electron microscope study of pyramidal neurons in the Ammon's Horn of the rabbit. J Anat 97:189-201.

Hansen PG, Meier E, Schousboe A (1984) GABA influences the ultrastructural composition of cerebellar granule cells during development in culture. Int J Dev Neurosci 2:247-257.

Harris KM, Kater SB (1994) Dendritic spines: cellular specializations imparting both stability and flexibility to synaptic function. Annu Rev Neurosci 17:341-371.

Haydon PG, McCobb DP, Kater SB (1984) Serotonin selectively inhibits growth cone motility and synaptogenesis of specific identified neurons. Science 226:561-564.

Hendry S, Carder RK (1992) Organization and plasticity of GABA neurons and receptors in monkey visual cortex. In: Progress in brain research, Vol 90 (Mize RR, Marc RE, Sillito AM, eds), pp 477-502. Amsterdam: Elsevier.

Hendry SHC, Jones EG (1986) Reduction in number of immunostained GABAergic neurons in deprived-eye dominance columns of monkey area 17. Nature 320:750-753.

Horner CH (1993) Plasticity of the dendritic spine. Prog Neurobiol 41:281-321.

Jessell TM (1988) Adhesion molecules and the hierarchy of neural development. Neuron 1:3-13.

Kaufman DL, Houser CR, Tobin AJ (1991) Two forms of the $\gamma$-aminobutyric acid synthetic enzyme glutamate decarboxylase have distinct intraneuronal distributions and cofactor interactions. J Neurochem 56:720-723.

Killisch I, Dotti CG, Laurie DJ, Lüddens H, Seeburg PH (1991) Expression patterns of $\mathrm{GABA}_{\mathrm{A}}$ receptor subtypes in developing hippocampal neurons. Neuron 7:927-936.

McCobb DP, Haydon PG, Kater SB (1988) Dopamine and serotonin inhibition of neurite elongation of different identified neurons. J Neurosci Res 19:19-26.

Michler-Stuke A, Wolff JR (1987) Facilitation of an inhibition of neurite elongation by GABA in chick tectal neurons. In: Neurotrophic activity of GABA during development (Redburn DA, Schousboe A, eds), pp 253-266. New York: Alan R. Liss.

Navone F, Jahn R, di Gioia G, Stukenbrok H, Greengard P, De Camilli P (1986) Protein p38: an integral membrane protein specific for small vesicles of neurons and neuroendocrine cells. J Cell Biol 103:2511-2527.

O'Leary DDM, Koester SE (1993) Development of projection neuron types, axon pathways, and patterned connections of the mammalian cortex. Neuron 10:991-1006.

Papa M, Bundman MC, Greenberger V, Segal M (1995) Morphological analysis of dendritic spine development in primary cultures of hippocampal neurons. J Neurosci 15:1-11.

Rall W (1970) Cable properties of dendrities and effects of synaptic location. In: Excitatory synaptic mechanisms (Andersen P, Jensen JKS, eds), pp 175-187. Oslo: Universitiets Forlaget.

Ramakers GJA, De Wit C, Wolters PS, Corner MA (1993) A developmental decrease in NMDA-mediated spontaneous firing in cultured rat cerebral cortex. Int J Dev Neurosci 11:25-32.

Ramakers GJA, van Galen H, Feenstra MGP, Corner MA, Boer GJ (1994) Activity-dependent plasticity of inhibitory and excitatory amino acid transmitter systems in cultured rat cerebral cortex. Int $\mathbf{J}$ Dev Neurosci 12:611-621.

Ready DF, Nicholls JG (1979) Identified neurons isolated from leech CNS make selective connections in culture. Nature 281:67-69.

Reetz A, Solimena M, Matteoli M, Folli F, Takei K, De Camilli P (1991) GABA and pancreatic $\beta$-cells: colocalization of glutamic acid decarboxylase (GAD) and GABA with synaptic-like microvesicles suggests their role in GABA storage and secretion. EMBO J 10:1275-1284.

Scholl DA (1953) Dendrite organization in the neurons of visual and motor cortices of the cat. J Anat 87:387-406.

Seress L, Frotscher M, Ribak CE (1989) Local circuit neurons in both the dentate gyrus and Ammon's horn establish synaptic connections with principal neurons in five day old rats: a morphological basis for inhibition in early development. Exp Brain Res 78:1-9.

Seress L, Ribak CE (1990) The synaptic connections of basket cell axons in the developing rat hippocampal formation. Exp Brain Res 81:500-508.

Shatz CJ, Stryker MP (1988) Prenatal tetrodotoxin infusion blocks segregation of retinogeniculate afferents. Science 242:87-89.

Shi Y, Veit B, Bœkkeskov (1994) Amino acid residues 24-31 but not palmitoylation of cysteines 30 and 45 are required for membrane anchoring of glutamic acid decarboxylase, $\mathrm{GAD}_{65}$. J Cell Biol 124:927-934.

Solimena M, Dirkx Jr R, Radzynski M, Mundigl O, De Camilli P (1994) A signal located within amino acids 1-27 of GAD65 is required for its targeting to the Golgi complex region. J Cell Biol 126:331-341.

Streit P (1984) Glutamate and aspartate as transmitter candidates for systems of the cerebral cortex. In: Cerebral cortex: functional properties of cortical cells (Jones EG, Peters A, eds), New York: Plenum.

Stryker MP, Harris WW (1986) Binocular impulse blockade prevents the formation of ocular dominance columns in cat visual cortex. J Neurosci 6:2117-2133.

Taylor J, Docherty M, Gordon-Weeks PR (1990) GABAergic growth cones: release of endogenous $\gamma$-aminobutyric acid precedes the expression of synaptic vesicle antigens. J Neurochem 54:1689-1700.

Tessier-Lavigne M (1994) Axon guidance by diffusible repellents and attractants. Curr Opin Genet Dev 4:596-601.

Uchizono K (1968) Axon identification in the cerebellar cortex of the cat. Arch Histol Jpn 29:399-424.

Van Huizen F, Romijn HJ, Habets AMMC (1985) Synaptogenesis in rat cerebral cortex cultures is affected during chronic blockade of spontaneous bioelectric activity by tetrodotoxin. Dev Brain Res 19:67-80.

Verderio C, Silvia C, Fumagalli G, Matteoli M (1994) Spatial changes in calcium signaling during the establishment of neuronal polarity and synaptogenesis. J Cell Biol 126:1527-1536.

Xie X, Smart TG (1991) A physiological role for endogenous zinc in rat hippocampal synaptic neurotransmission. Nature 349:521-524.

Zanka Z, Jones DG (1982) Junctions in rat neocortical explants cultured in TTX-, GABA-, and $\mathrm{Mg}^{++}$- environments. Brain Res Bull 8:273-278.

Zhang L, Spigelman I, Carlen PL (1991) Development of GABAmediated, chloride-dependent inhibition in CA1 pyramidal neurones of immature rat hippocampal slices. J Physiol (Lond) 444:25-49.

Zoran MJ, Doyle RT, Haydon PG (1990) Target-dependent induction of secretory capabilities in an identified motoneuron during synaptogenesis. Dev Biol 138:202-213. 